\title{
Infra-Diaphragmatic Gray Zone Lymphoma Presenting with Paraneoplastic Neurological Syndrome
}

\author{
Torres J 1,4 Hawkes EA ${ }^{1,2,3 *}$, Hosking $P^{1}$ and Bladin \\ $\mathbf{C}^{1,2}$ \\ ${ }^{1}$ Department of Oncology, Eastern Health, Australia \\ ${ }^{2}$ Monash University, Australia \\ ${ }^{3}$ Olivia Newton J ohn Cancer Centre, Austin Hospital, \\ Australia \\ ${ }^{4}$ Goulburn Valley Health Australia \\ *Corresponding author: Hawkes EA, Department of \\ Oncology, Eastern Health, 8 Arnold St, Box Hill, Victoria, \\ Australia
}

Received: May 02, 2017; Accepted: May 31, 2017;

Published: June 22, 2017

\section{Case Presentation}

A previously healthy 57-year-old man presented to the emergency department with three weeks of progressive urinary retention and rapidly progressive bilateral lower limb weakness causing decreased mobility, on a background of $15 \mathrm{~kg}$ ' weight loss over two months. $\mathrm{He}$ denied any infective symptoms, headache or neck stiffness.

The patient was a current smoker and consumed excess alcohol but had no significant medical conditions and no regular medications. Family history included colorectal cancer, in his father, aged 70 [1-3].

Neurological examination revealed bilateral lower limb weakness in an upper motor neuron muscle distribution, hypertonia, hyperreflexia, bilateral spontaneous clonus, and up-going plantar responses consistent with spastic paraparesis. Sensation remained intact.

Initial investigations revealed normal full blood examination, biochemistry, lactate dehydrogenase, C-reactive protein, Erythrocyte Sedimentation Rate, vitamin B12 and folate. The gammaglutamyltransferase and alanine aminotransferase were elevated, (230 IU/L [ULN: 71] and ALT 118 IU/L [ULN: 40] respectively). HIV and syphilis serology were negative. Magnetic resonance imaging (MRI) of brain and whole spine excluded a space-occupying lesion at any level, intracranial demyelization, spine infiltration and myelitis.

Cerebrospinal fluid (CSF) analysis revealed elevated lymphocytes $\left(15 \times 10^{6} / \mathrm{L}\right)$ but normal polymorph counts and biochemistry, negative culture and cytology. Flow-cytometry failed to reveal abnormal clonal population. Nerve conduction studies were also unremarkable. Paraneoplastic-associated antibodies including anti-Yo, anti-CV2, anti-Glutamic Acid Decarboxylase, anti-Hu and anti-Ri were all negative. Computer Tomography (CT) of neck, chest, abdomen, pelvis demonstrated only prominent left iliac lymph nodes $(<10 \mathrm{~mm})$ but no other abnormalities (Figure 1B).

During admission, a significant progression of the spastic paraparesis ensued and left-sided internuclear ophthalmoplegia developed. A repeat MRI of brain and spinal cord was done but

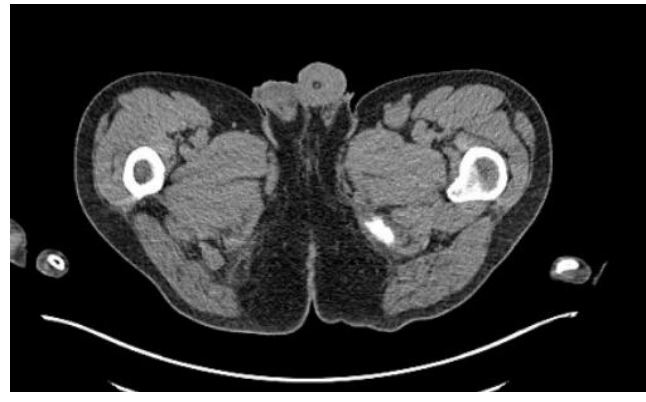

Figure 1A: CT demonstrating prominent $1 \mathrm{~cm}$ left inguinal lymph nodes. No other findings were identified.

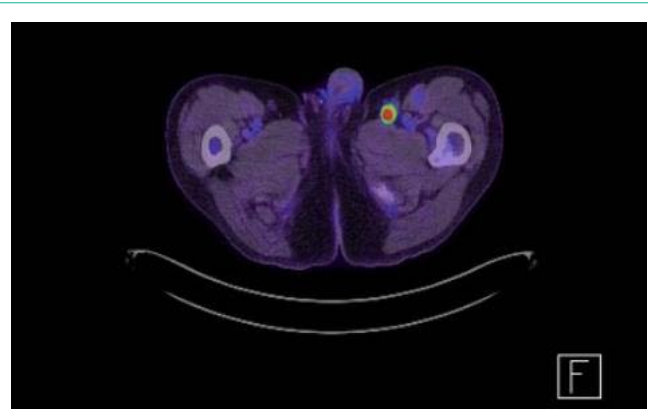

Figure 1B: Initial FDG PET-CT revealing intense FDG lymph node in the medial aspect of left inguinal area.

remained unremarkable.

Given the ongoing, rapid neurological deterioration despite administration of high-dose corticosteroids, a positron emission tomography (FDG-PET) was performed to exclude occult malignancy. Intensely FDG-avid lymph nodes were identified in the left abdominal para-aortic and inguinal regions (Figure 1B). An urgent excisional inguinal node biopsy revealed multiple poorly circumscribed nodules containing large atypical cells with large pleomorphic nuclei and vacuolated cytoplasm without classical ReedSternberg cells and immunophenotyping with positive CD20 and
Ann Hematol Oncol - Volume 4 Issue 6 - 2017

ISSN : 2375-7965 | www.austinpublishing group.com

Hawkes et al. () All rights are reserved
Citation: Torres J, Hawkes EA, Hosking P and Bladin C. Infra-Diaphragmatic Gray Zone Lymphoma Presenting with Paraneoplastic Neurological Syndrome. Ann Hematol Oncol. 2017; 4(6): 1157. 


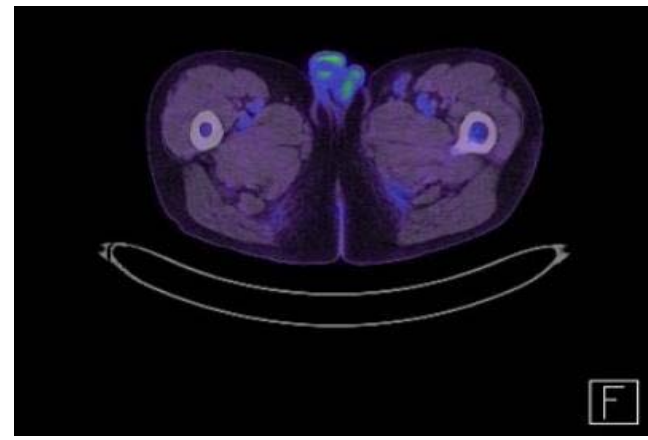

Figure 1C: FDG PET-CT revealing complete metabolic response of lesion on left inguinal area after six cycles of chemotherapy with Rituximab-Adriamycin, Bleomycin, Vinblastine and Dacarbazine.

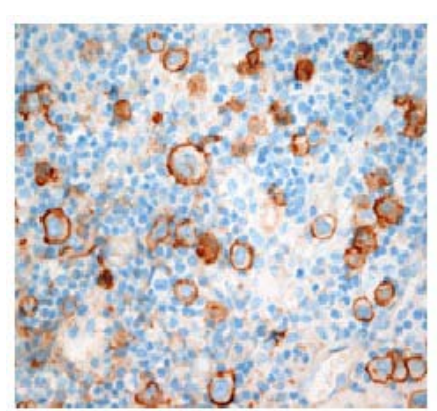

CD30 magnification $400 x$

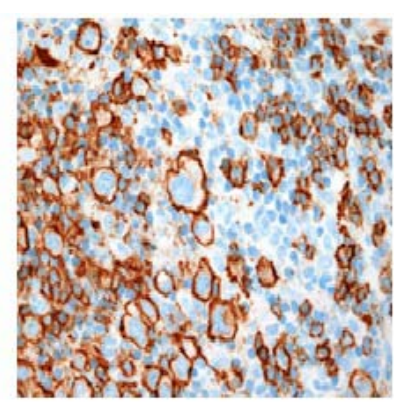

CD20 magnification $400 x$
Figure 2: Lymph node biopsy material revealing Gray Zone Lymphoma. Multiple poorly circumscribed nodules containing moderate numbers of large atypical cells with large vesicular pleomorphic nuclei, vacuolated cytoplasm with scattered multilobated cells but without classical Reed-sternberg cells. Immunophenotyping showed a mixed staining pattern with some markers favouring $\mathrm{CHL}$ (CD30 strongly diffusely positive, CD15 focally positive in some tumour cells and CD45 negative) but also positive staining for B-cell non-Hodgkin lymphoma (CD20, Pax5 and CD79a strongly diffusely positive).

PAX5, strongly positive CD30 and negative CD15 consistent with GZL (Figure 2).

R-ABVD chemotherapy (rituximab, adriamycin, bleomycin, vinblastine, dacarbazine) was commenced urgently. Complete resolution of opthalmoplegia and significant improvement of gait occurred after the first cycle. After 2 cycles (4 treatments), all neurological Symptoms and signs was resolved. Restaging PET after 3 and 6 cycles demonstrated a complete metabolic response at all sites (Figure 1C). The patient has been on surveillance for 12 months and remains entirely well.

In summary, we present an atypical presentation of infradiaphragmatic GZL presenting with non-enlarged PET-avid lymphadenopathy but a paraneoplastic spastic paraparesis that completely resolved after starting chemotherapy.

\section{Discussion}

GZL is a recognised entity in the WHO classification of haematological malignancies [3] as B-cell lymphoma with features intermediate between DLBCL and cHL. As suggested by gene expression profiling studies, GZL is an overlap of primary mediastinal B-cell non-Hodgkin lymphoma (PMBCL) and nodular-sclerosingc HL with pathological features that are transitional between these two diseases [4]. Typically, GZL presents with an enlarging, symptomatic mediastinal mass and/or supra-diaphragmatic lymphadenopathy. Immunohistochemical analysis reveals markers for both DLBCL (CD20+, CD19+, PAX5+) and cHL (CD15+, CD30+) [4].

This case highlights the important role of PET in diagnosing occult malignancy, including aggressive lymphomas, in the absence of CT findings, where there is strong clinical suspicion [5]. One series reported a high negative predictive value of combined modality PETCT, but the study did not report the rate of PET-positivity in those with a normal CT [6]. Another study showed limited diagnostic value of PET as screening tool to detect occult malignancy [7].

In clinical practice, we often use PET to assess avidity in suspicious lesions identified on CT to evaluate the malignant potential. However, our case supports the argument that PET scanning should be part of a diagnostic work up for patients presenting with findings suspicious of PNS, even with a normal CT, as it can assist with early diagnosis and allow PET-directed biopsy in a time-critical case such as ours.

It also demonstrates the potentially reversible nature of PNS which are generally thought to not to improve dramatically after achieving tumour control [2]. Early treatment in these patients is crucial to recovery of their clinical condition.

In summary, PET should be considered part of the diagnostic process for clinical scenarios suspicious for occult malignancy. GZL can present as a PNS and with infra-diaphragmatic disease. Prompt histological diagnosis and treatment, as illustrated by this case, can lead to complete resolution of the disease and the PNS.

\section{References}

1. Grimm S, Chamberlain M. Hodgkin's Lymphoma: A review of Neurologic Complications. Advances in Hematology. 2011.

2. Blaes F, Tschernatsch M. Paraneoplastic neurological disorders. Expert Review of Neurotherapeutics. 2015; 15: 187-193.

3. Swerdlow SH, Campo E, Harris NL, Jaffe ES, Pileri SA, Stein H, et al. World Health Organization Classification of Tumours of Haematopoietic and Lymphoid Tissues. IARC Press, Lyon 2008.

4. Dunleavy k and Wyndham H. Wolson. Primary mediastinal B-cell lymphoma and mediastinal gray zone lymphoma: do they require a unique therapeutic approach? Blood. 2015; 125: 33.

5. Fan HB. Use of (18) F-FDG PET/ET to locate primary malignancies in patients with hepatic cirrhosis and malignant ascites. Chin J Cancer Research. 2013; 25: 500-504.

6. Kristensen SB, Hess S, Petersen H, Hoilund-Carlsen PF. Clinical value of FDG-PET/CT in suspected paraneoplastic syndromes: a retrospective analysis of 137 patients. Eur J Nucl Med Mol Imaging. 2015; 42: 2056-2063.

7. Chauchard M, Benali K, Papo T, Sacre K. Positron emission tomography combined with computed tomography as a screening tool for occult malignancy in patients with unprovoked venous thromboembolism: an observational study. Medicine (Baltimore). 2014; 93: e110. 